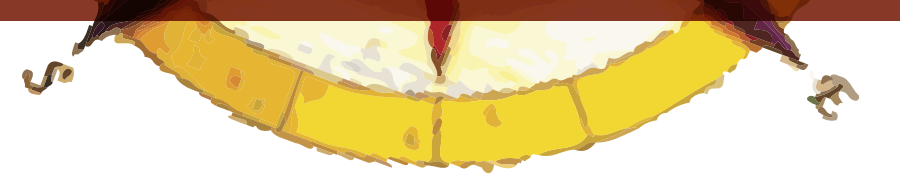

\title{
REVISIÓN EPISTEMOLÓGICA A LA DIDÁCTICA DE LA GEOGRAFÍA. CONTRIBUCIÓN CURRICULAR Y METODOLÓGICA
}

\author{
Alfonso García De La Vega ${ }^{17}$
}

\section{RESUMEN}

El discurso epistemológico de una disciplina académica se descompone en diversos elementos. En este trabajo, en primer lugar, se revisan la transposición didáctica, la disciplinariedad, los conocimientos didácticos de contenido y la reflexión profesional del docente, que constituyen cuatro paradigmas en el marco de la discusión educativa de las didácticas específicas. A continuación se extraen las aportaciones más significativas de cada una ellas a la didáctica de la Geografía. Por último, se plantea algunas cuestiones referidas al currículo y al enfoque metodológico como posibles vías de aproximación significativa, aunque parcial, al desarrollo epistemológico de la didáctica de la Geografía.

Palabras clave: transposición didáctica, disciplinariedad, conocimiento didáctico de contenido, profesional reflexivo, epistemología, didáctica de la Geografía.

\section{RESUMO}

O discurso epistemológico de uma disciplina acadêmica compreende várias categorias. Este trabalho tem como objetivo passar por quatro paradigmas do atual debate educacional no ensino de áreas específicas, nomeadamente a transposição didática, interdisciplinaridade, conhecimento pedagógico do conteúdo e da prática profissional reflexiva. Então, conclue-se pelas contribuições mais siginificativas de cada paradigma para o ensino de geografia. Finalmente, aborda-se algumas questões relacionadas com o currículo e ao enfoque metodológico como vias possíveis para se aproximar, de forma significativa, mas parcial, para o desenvolvimento epistemológico do ensino de Geografia.

Palavras-chave: transposição didática,disciplinaridade, conhecimento pedagógico do conteúdo, profissional reflexiva, epistemologia, ensino de geografia.

17 Universidad Autónoma de Madrid.

Agradecimientos a Esther Andrés por la traducción del resumen al inglés. 


\begin{abstract}
The epistemological discourse of an academic discipline comprises various categories. This paper aims at going through four paradigms of the current educational debate in the teaching of specific subject areas, namely, didactic transposition, disciplinarity, pedagogical content knowledge and reflective professional practice. Then, it is drawn the most outstanding contributions by each paradigm to geography teaching. Finally, some issues are tackled related to the curriculum and the methodological approach as possible thoroughfares for coming closer, in a significant but partial way, to the epistemological development of Geography teaching.
\end{abstract}

Key-words: Didactic Transposition, Disciplinarity, Pedagogical Content Knowledge, Reflective Professional Practice, Epistemology, Geography Teaching.

\title{
CONFLICTO EPISTEMOLÓGICO EN LA DIDÁCTICA DE LAS DISCIPLINAS
}

Audigier (1992, p.18) señalaba el doble objetivo de la didáctica de la Geografía: por un lado analizar y entender el proceso de enseñanza y aprendizaje, y por otro, proponer las herramientas para mejorar dicho proceso. Callai (2002, p. 93) expresa que el profesor precisa dominar tanto el proceso de enseñanza-aprendizaje como conocer los contenidos curriculares de su trabajo.

La formación y consolidación de una estructura epistemológica en las didácticas específicas ha estado influida por los paradigmas provenientes de otras materias. Ajenas a la disciplina, estos paradigmas han generado confusión en el corpus geográfico escolar. Sin tratar de agotar la discusión, a continuación se exponen los principales rasgos de estos paradigmas y su aportación a la didáctica de la Geografía.

\section{Transposición didáctica y disciplinariedad}

Rodríguez Lestegás (2000, p. 109) expresó la divergencia conceptual de los paradigmas formulados por Chevallard (1991), la transposición didáctica, y Chervel (1988), la disciplinariedad. E1 primer autor persigue la proximidad adaptada de la ciencia en la didáctica, en cuanto que se legitimiza por hallarse suficientemente próximo a ella. Por el contrario, la disciplinariedad resalta el valor intrínseco del currículo escolar, que como transmisión de conocimientos, constituye el bagaje cultural que representa el referente común a todos los ciudadanos.

Verret acuñó el término de transposición didáctica, según afirman Chervel $(1988$, p. 67) y Perrenoud (1998, p. 489). Chevallard (1991) aplicó este concepto, de naturaleza sociológica, al campo de las matemáticas. Este autor se refiere a los contenidos académicos que, modificados y adaptados a la edad del alumnado, se integran en el proceso de enseñanza-aprendizaje.

En sus palabras, "todo proyecto de enseñanza y de aprendizaje se constituye dialécticamente con la identificación y la designación de unos contenidos de saberes como contenidos a enseñar" (Chevallard, 1991:45). Este mismo autor consideró que, bajo el prisma de las matemáticas, existen unas "prácticas sociales en matemáticas" que definió como los ámbitos pragmáticos donde se generan aprendizajes matemáticos (Chevallard, 1991, p. 174). 
Reboul (1991, p. 112) elaboró una analogía entre la retórica y la transposición didáctica. Por cuanto que, según este autor, el profesor debe atraer y mantener la atención del alumnado ilustrando los conceptos, facilitando el recuerdo y motivando hacia el esfuerzo. En definitiva, como recalca el citado autor, "el docente proporciona claridad y coherencia a su disciplina que no tiene necesariamente en tanto que ciencia".

Chervel (1988, p. 117) manifestó que las disciplinas constituyen la historia cultural de la sociedad, aunque muchas de ellas se hayan definido como tales en el transcurso del siglo pasado. Y, el conocimiento escolar se sustenta sobre el entramado de estas disciplinas, proporcionando una cultura específica al alumnado.

La diferencia existente entre el conocimiento geográfico científico y escolar ha sido descrita por Rodríguez Lestegás $(2007$, p. 528). De manera que en el primero la ciencia constituye el saber a medida que se van produciendo y alcanzando los logros de las investigaciones y éstas transfieren dicho conocimiento a la sociedad. En el segundo, el conocimiento escolar, se nutre de los resultados consolidados de aquellos conocimientos científicos.

En suma, estos dos paradigmas educativos muestran un cierto antagonismo, al tiempo que expresan un grado de complementariedad (García De La Vega, 2009, p.11). Ahora bien, si la transposición didáctica se ha llegado a mostrar en las matemáticas desde hace décadas, e incluso, en otras áreas experimentales, como la física, no parece tan evidente la aplicación en la didáctica de la Geografía.

Sin embargo, podría considerarse la justificación, la valoración y la aportación a la didáctica de la Geografía de ambos paradigmas, pues según Oliveira (2011, p. 61): "A linha da história das disciplinas escolares é fundamental por que situa o contexto sócio-histórico de origem, desenvolvimento e constituição dos saberes escolares [...]. A transposição didática, por sua vez, oferece as bases para a compreensão da estruturação lógica do saber escolar".

\section{Reflexión docente y Geografía}

Cabe señalar, que para presentar las aportaciones de la reflexión docente, Perrenoud (1998 p.505) ofrece una perspectiva disciplinar más amplia y elaborada sobre la transposición didáctica. Este autor considera que el recorrido transpositivo comienza en las prácticas profesionales. Para ello, se ha de describir e identificar las citadas prácticas que, según Perrenoud, conducen a definir los rasgos de las competencias formativas.

En este sentido, la necesidad de registrar los rasgos identificativos de las prácticas ofrece diferencias significativas entre los distintos ámbitos profesionales. En el extremo opuesto, Chervel (1988, p.106) afirma que "les practiques pédagogiques se sont-elles montrées efficaces ? Rares sont les historiens de l'enseignement qui ont soulevé le problème". En el otro extremo, Schön (1998:186 y ss.) formuló la práctica reflexiva de la actividad del arquitecto dedicado al planeamiento urbano, destacando los hitos reformuladores para la reflexión profesional.

Ahora bien, si se llevara al campo de la docencia, el registro y la evaluación de dichas prácticas proporcionarían los datos precisos para identificar y valorar las competencias profesionales. Así, según se expuso más arriba, la práctica reflexiva ha definido una línea de investigación educativa, 
procedente de otro ámbito profesional, la arquitectura. De hecho, Schön (1992, p. 267 y ss.) propuso renovar la fórmula habitual del prácticum profesional para conferir un mayor grado de reflexión en la práctica habitual de toda profesión. Perrenoud $(2004$, p. 12) propondría la práctica reflexiva como un paradigma educativo integrador y reflexivo.

Las dos aportaciones más relevantes de este paradigma, y que pudieran interesar a la didáctica de la Geografía, son el habitus y la indagación. El habitus, según Bourdieu (en Perrenoud, 2004, p. 79) viene a ser "el sistema de estructuras de pensamiento, de percepción, de evaluación y de acción de nuestras prácticas". Si bien, según aclara el propio autor, no se refiere exclusivamente a las prácticas profesionales, si no también a las cotidianas. Brubacher, Case y Reagan (2005, p. 54) consideran a la indagación como "los intentos por conocer y dar sentido al mundo que nos rodea".

Si la reflexión en la práctica profesional supone una revisión de todas las rutinas e innovaciones educativas llevadas a cabo en el proceso de enseñanza-aprendizaje, la indagación, como se verá más adelante, conduce a elaborar una estrategia didáctica vinculada a diversos enfoques metodológicos descritos más adelante (proyecto de trabajo y aprendizaje basado en problemas).

\section{Los conocimientos didácticos/pedagógicos de contenido}

Seguramente, la proposición de Shulman $(2005$, p.11) sobre los denominados "conocimientos didácticos de contenido", o también "conocimientos pedagógicos del contenido", aviva la discusión de la didáctica en las disciplinas. En este sentido, tal vez, esto permita elaborar un paradigma educativo que, quizá, impregne profundamente la perspectiva epistemológica en la didáctica de la Geografía.

Shulman (2005, p. 11) menciona los siguientes tipos de categorías vinculadas a los conocimientos del docente: del contenido propiamente, de la didáctica general, del currículo, de la didáctica del contenido, de los alumnos y sus características, de los contextos educativos y, por último, de los objetivos, finalidad y valores educativos y fundamentos históricos y filosóficos de la educación. A este elenco prolijo, Gudmundsdóttir y Shulman $(2005$, p. 2) añaden el "conocimiento de los contextos, marcos y gestión educativa".

Y, el propio Shulman (2005, p. 11) resalta las fuentes de conocimiento como base de la enseñanza: la formación académica de la disciplina a enseñar, los materiales y el contexto del proceso educativo, la investigación sobre la escolarización y otros fenómenos socioculturales y, por último, la sabiduría en la práctica docente.

Shulman $(2005$, p. 12) afirma que "el profesor es miembro de una comunidad académica. Debe comprender las estructuras de enseñanza, los principios de la organización conceptual, así como los principios de indagación". En este mismo sentido, este autor cita a Schwab (1964), quien definió "conocimiento de estructuras sustantivas" a aquellas ideas y destrezas en el ámbito del saber. Y, con el "conocimiento de estructuras sintácticas" se refiere a la incorporación de nuevas ideas y a la eliminación de las ideas incorrectas, carentes o superadas.

Sin embargo, al igual que sucede en la transposición didáctica, hay autores dedicados al estudio de las matemáticas que han revisado las ideas propuestas por Schulman (cfr. BALL, THAMES y PHELPS, 2008). En este sentido, tal vez, la didáctica de la Geografía necesite una revisión detenida de esta aportación pedagógica. 


\section{LA SUPERACIÓN DEL PARADIGMA DISCIPLINAR EN LA DIDÁCTICA DE LA GEOGRAFÍA}

A lo largo de la historia, los paradigmas académicos han jalonado el devenir epistemológico de las todas las disciplinas, generando numerosas controversias entre las escuelas de pensamiento. E1 carácter pluriparadigmático de la Geografía ha influido en el diseño curricular en la escuela, aunque dicho rasgo distintivo se ha consolidado en la Geografía. Unwin (1995, p. 41) considera que tanto para comprender su valor actual como para saber las posibilidades de cambio social, resulta esencial conocer el pasado de una disciplina.

El conocimiento de la historia de la disciplina, afirma Unwin, nos conduce a los límites de la comprensión de la realidad disciplinar. Por tanto, hay que poseer un conocimiento disciplinar y profesional para desarrollar como docente las estructuras de conocimiento, arriba mencionadas por Schwab, esto es, sustantivas y sintácticas.

Sin embargo, los sucesivos paradigmas geográficos han transferido sus postulados a los desarrollos curriculares, suscitando controversias epistemológicas en la didáctica. Pues el corpus de los elementos curriculares (objetivos, contenidos, competencias básicas y criterios de evaluación) ha divagado según los enfoques epistemológicos de dichos paradigmas.

Audigier (1992, p. 20-21) distingue cuatro características en la Geografía escolar: el conjunto de conocimiento universales, los métodos de enseñanza, los procedimientos de evaluación comunes y los sistemas de motivación destinados a participar a los alumnos en el proceso de enseñanzaaprendizaje. Seguramente, estas características y su combinación podrían constituir una parte de los elementos, mencionados al comienzo de este trabajo, como el corpus epistemológico de la didáctica de la Geografía.

En este sentido, la investigación en didáctica de la Geografía debiera abrirse a una discusión exenta de prejuicios y dualismos. Y, si cabe, al margen de los paradigmas ajenos a la propia didáctica, atendiendo la complejidad de la realidad y formulando un discurso epistemológico propio. Tal como se expresa Cavalcanti (2011, p. 196):

"Na medida em que compreende-se que o real é complexo, composto por elementos subjetivos e objetivos, naturais e sociais, materiais e imateriais, o caminho do discurso geográfico é no sentido de aprender as inter-relações entre esses elementos, sem dicotomias".

Si se abandonan tanto los paradigmas de la propia disciplina y como las injerencias procedentes de otras disciplinas, quizá se podría replantear algunas cuestiones clave de la didáctica de la Geografía. Entre estas cuestiones, algunas se abordan en el siguiente apartado, aún quedan por afrontar otras perspectivas que vayan consolidando el corpus de conocimiento de la didáctica en Geografía. Souza (2011 p.125) confronta dos perspectivas en la construcción del conocimiento geográfico, los obstáculos epistemológicos de Bachelard (2003) y el conocimiento voluntario de Vygotsky (2010).

En suma, la tendencia a futuro de los estudios sobre la didáctica de la Geografía debiera considerar la elaboración de un corpus que canalice los esfuerzos desde todas las perspectivas posibles. Esto quiere decir que además de los enfoques epistemológicos, también se deben considerar las aportaciones referidas a los elementos curriculares, a las secuencias de contenidos, a la metodología y a los 
recursos didácticos. A continuación se abordan algunos de estos aspectos, sin ánimo de agotarlos se pretende esbozar algunas líneas de trabajo sobre los contenidos, la innovación de los enfoques metodológicos y sus experiencias y la renovación de los recursos didácticos.

\section{ENTRE LOS ELEMENTOS CURRICULARES... LOS CONTENIDOS GEOGRÁFICOS}

En el marco educativo, salvo la novedad de las competencias básicas, los contenidos constituyen la estructura organizativa del currículo. Ahora bien, ante las ideas arriba expuestas, García Pérez (2011, p.10) resalta el esfuerzo del debate curricular por conocer el paradigma que se identificaba mejor con la geografía escolar.

De hecho, los contenidos podrían estructurarse según las propuestas curriculares de rasgos de naturaleza geográfica, s. 1., o sociales (Herrero Fabregat, 1995, p. 137-146). O, incluso, se podrían sistematizar las prácticas cotidianas de las personas, según las sugerencias de Cavalcanti $(2008$, p. 20).

Gurevich (2006, p.64) afirmó que "la geografía de la escuela es la geografía de finales del siglo XIX y principios del XX, entendida como la ciencia de los lugares [...] Su interés radica en identificar y pormenorizar aspectos y atributos de cada porción de la superficie terrestre. Se atiende al criterio de lo único, de lo que tiene de excepcional cada lugar, sin posibilidad de realizar articulaciones y generalizaciones que permitan transferir esos conocimientos a otros contextos".

La enumeración de datos (p. e., los accidentes geográficos) y la adquisición de conceptos complejos (p. e., la rotación y la traslación de la tierra) conformó un diseño curricular geográfico convertido en clásico. Por ello, interesa aplicar otro tipo de estructura curricular asociada al aprendizaje de situaciones reales. Y, por consiguiente, desarrollar estrategias cognitivas, destrezas manuales y habilidades sociales (García De La Vega, 2010b y en prensa).

\section{La integración del currículo en Geografía}

Aisenberg (1998, p. 50) definió "contenidos pertinentes" como todos aquellos que pueden favorecer mejor el aprendizaje significativo del alumnado en dirección al objetivo establecido. Beane (2005) propuso un currículo integrado, mediante una estructura de los elementos disciplinares. En cierta manera, lo que se propone aquí es el paisaje como eje de los contenidos de un currículo integrado e interdisciplinar. De manera que la Geografía se convertía en la materia aglutinadora de las demás disciplinas escolares.

De hecho, tal como se expresa Beane (2005, p. 69) el currículo integrado supone la implementación de una secuencia de contenidos pertinentes desde las diferentes materias. Así, se persigue la complementariedad de los contenidos específicos de cada disciplina a través de la elaboración de unos proyectos de trabajo que asimilen todos los elementos curriculares.

Desde la didáctica de la Geografía se pueden proponer numerosos temas nucleares que conciernen a las actuaciones humanas en el territorio, no solamente sobre la naturaleza. También la gestión medioambiental y sus implicaciones sociales, económicas y culturales. Por consiguiente, la sostenibilidad, la preservación de la naturaleza y la valoración de las actuaciones humanas sobre los paisajes componen el entramado de estos temas nucleares (García De La Vega, 2011). 
Bajo la misma óptica metodológica, Coll (1997, p. 153) observa la necesidad de confeccionar un currículo integrado, que recoja todo tipo de informaciones y distinto tipo de fuentes. Además, conviene integrar los procesos de aprendizaje y el desarrollo psicoevolutivo del alumno. En este sentido, según afirma Camilloni (1998, p. 185):

"Para construir conocimientos en el campo de las ciencias sociales, el alumno necesita desarrollar sus competencias en la aplicación, enriquecimiento, profundización de conceptos y teorías, así como adquirir destrezas y habilidades cognitivas y actitudes positivas".

Chomsky (2001, p. 10) ironiza sobre el compromiso social de los maestros, pues éstos nunca piden a sus alumnos el análisis de las estructuras sociopolíticas. Y, afirma, que los maestros tienen que ser críticos y denunciar la injusticia social y los sistemas de miseria. Tal vez, alcanzar un mayor grado de compromiso social en la educación suponga la elaboración y la organización de un nuevo planteamiento curricular.

La propuesta de Fernández Caso (2007, p. 30-31) recoge tres criterios orientadores del currículo: significatividad lógica y epistemológica, relevancia social, significatividad psicológica. Una propuesta que integraría las secuencias de contenidos geográficos sobre los mencionados criterios y proporcionaría un currículo integrado y articulado sobre dichos contenidos.

En la Geografía y en las ciencias sociales, se desarrolla una empatía desde el conocimiento y el análisis preciso de la realidad. Estos análisis convergen en la interpretación de los escenarios geográficos y, como consecuencia, la formación del pensamiento crítico y el compromiso social que generan los valores cívicos y éticos del individuo.

\section{Y de los contenidos... ¿sólo los conceptos?}

Con el fin de desarrollar un currículo integrado e interdisciplinar centrado en temas nucleares extraídos de la realidad, la didáctica de la Geografía precisa una jerarquía conceptual. Dicha jerarquía necesita definir unas determinadas categorías geográficas, conforme a determinados criterios de discriminación. Así, por ejemplo, estos criterios pudieran corresponder a la escala local y global en la ordenación del territorio y el registro del nivel de actividad humana en las transformaciones del paisaje. Y, de la misma manera se deben definir, por ejemplo, el rango de los elementos de relieve.

Vilhena de Moraes (2010, p. 101) propone el aprendizaje por proposición, definido como una de las tres categorías del aprendizaje significativo de Ausubel, para desarrollar una jerarquía conceptual en la Geografía. Esta misma autora ofrece tres procedimientos desde el aprendizaje significativo: subordinado, supraordenado y combinatorio.

Esta organización cognitiva se muestra muy próxima al mapa conceptual de Novak. Incluso, este mismo autor considera la aportación personal a la "propia jerarquía conceptual idiosincrática" (Novak, 1988, p. 35). Y, estas aportaciones se vinculan a la creatividad y a la integración de nuevos conceptos y la adaptación de los mismos en las redes conceptuales, generando nuevas interrelaciones en los mapas conceptuales.

Cavalcanti (2010, p. 26) señala, siguiendo a Vygotsky, que la memoria y la asociación, por sí solos, no propician la formulación de conceptos. La identificación de los rasgos característicos de un 
concepto permite aproximarse a la definición del mismo. Vygotsky (2010, p. 213) afirma que este paso exige una experimentación, pues el desarrollo de los conceptos no es un proceso de asimilación directa e inmediata.

Desde el enfoque constructivista, los rasgos dominantes de un proceso de enseñanza-aprendizaje deben formularse a partir del impulso de ciertas estrategias didácticas vinculadas a los postulados próximos a dicho enfoque metodológico constructivista. Así, Vygotsky (2010, p. 213) afirma que la comprensión del desarrollo de los conceptos científicos en el niño permite elaborar métodos eficaces.

En la didáctica de la Geografía, Cavalcanti (2008, p. 88) insiste que los conceptos constituyen un lenguaje propio y, por tanto, se requiere dominar estos conceptos para analizar los fenómenos geográficos. Por ello hay que proporcionar un escenario educativo que permita desarrollar un proceso de enseñanza-aprendizaje donde los docentes y los alumnos establezcan un compromiso sobre el aprendizaje y sobre el papel a desarrollar por cada uno de ellos.

Evidentemente, las habilidades memorísticas pueden ser utilizadas para aplicarse a situaciones reales. Sin embargo, "la educación superior", sentencia Unwin (1995, p. 285), "no debe ocuparse de inculcar hechos aceptados, sino de capacitar a los estudiantes para que desarrollen sus propios enfoques críticos ante el mundo en que viven". En definitiva, se trata de transmitir una formación crítica y el "deseo de adquirir conocimientos emancipadores", como afirma este mismo autor.

Por consiguiente, no sólo se precisa establecer una categoría en los conceptos geográficos. También se puede transmitir a los alumnos la necesidad de generar con ellos, atendiendo a su edad, unos criterios comunes, que permitan delimitar los elementos y hechos geográficos. Esto es, ¿supone lo mismo hablar de monte y montaña?.

\section{EL ENFOQUE METODOLÓGICO. UNA VARIABLE MÁS EN LA EPISTEMOLOGÍA DE LA DIDÁCTICA}

El escenario educativo constituye el espacio donde se encuentran todos los agentes y los recursos educativos y sus interrelaciones. El enfoque metodológico de la didáctica de la Geografía correspondería al constructivismo que, según Benejam (1992, p. 41), ofrece una integración personal y social del aprendizaje. Por tanto, el constructivismo, siendo un paradigma, engloba un conjunto de postulados trabados entre sí, ofreciendo una perspectiva metodológica. El aprendizaje por descubrimiento de Bruner, el aprendizaje significativo de Ausubel, el aprendizaje entre iguales y la interacción social, así como la zona de desarrollo próximo, formulados por Vygotsky y, si cabe, se podría añadir las inteligencias múltiples de Gardner, como las principales aportaciones a este enfoque metodológico.

De este modo, según advierte Libâneo (1992, p. 6), constituye "una situación de enseñanzaaprendizaje como actividad conjunta y compartida entre el profesor y los alumnos. [...]. Y además, el alumno también elabora sus conocimientos, sus métodos de estudio y su afectividad". Stauffacher et al. (2006:255-256) resaltan el valor del alumno, tanto en su propio aprendizaje como en la organización y dinámica del grupo.

El planteamiento metodológico de determinados enfoques didácticos supone una interpretación de la realidad geográfica. Esto conduce a analizar los problemas socioterritoriales, explicar la ges- 
tión medioambiental y descubrir los nuevos escenarios urbanos, rurales y naturales, según se proponen Fernández Caso (2011) y Gurevich (2007).

Para ello, es preciso, en primer lugar, conocer y dominar las técnicas y métodos básicos de la Geografía para la posterior interpretación atinada de los hechos geográficos, donde se valoren las actuaciones humanas en el territorio. En la línea planteada por Shulman (2005), referido al conocimiento didáctico del contenido, por cuanto, dominar el discurso, el currículo, la didáctica y los recursos didácticos. Por tanto se propone una organización integrada del currículo, en torno a determinados temas nucleares interdisciplinares.

\section{Los proyectos de trabajo y la indagación en los contenidos geográficos}

A fin de establecer el papel de reto al problema planteado en la propuesta educativa se aproximan los contenidos a escenarios geográficos reales, reforzando el proceso de enseñanza-aprendizaje. En dicho proceso, el rasgo de mayor relevancia se refiere a la interdisciplinariedad, próxima a las posibilidades didácticas en el desarrollo de las competencias básicas de las materias, en cuanto que la Geografía es disciplina de síntesis.

En esta línea, García Pérez (2011, p. 11) manifiesta que la Geografía se podría articular en torno al tratamiento de problemas sociales y ambientales relevantes. En este sentido, los proyectos relacionados con el paisaje han afrontado problemas en los ecosistemas brasileños (Saito, 2006), conceptos clave de estudio (Batllori y Serra, 2010) o la lectura de paisajes mediante objetivos, instrumentos y criterios de evaluación (Castiglioni, 2010).

Este mismo enfoque de los contenidos, a través de las situaciones-problema, se podría abordar mediante proyectos de trabajo. Los textos literarios proporcionan un hilo conductor en la elaboración de estos proyectos, tanto por el refranero relacionado con el clima (Marrón Gaite, 2011) como por la producción de textos relacionados con los mitos y el paisaje (García De La Vega, 2010a, 2011 y en revisión).

El barrio, como estudio del entorno más próximo a la escuela, puede convertirse tanto en un proyecto de trabajo como en un proyecto educativo de centro de carácter interdisciplinar (Castelar, 2011, p. 88 y ss.). Si, en el primer caso, el proyecto puede acoger los contenidos de un curso o ciclo, en el segundo caso, el proyecto educativo puede convertirse en el eje interdisciplinar de varias etapas, e incluso, de todo el centro educativo. En este caso, debiera recogerse en un documento prescriptivo del centro, que comprometa a todo el claustro en la propuesta educativa.

Los itinerarios geográficos también se pueden convertir en un proyecto de trabajo, o bien, un proyecto educativo (García De La Vega, 2009). Los itinerarios didácticos en un centro poseerían un enfoque interdisciplinar entre las distintas áreas y generaría una posible secuencia de contenidos sobre un currículo integrado. Además, los itinerario fomentan la formación del pensamiento crítico para la adquisición de valores referidos al patrimonio natural y cultural (García De La Vega, 2004 y Giné Serrano, 2007).

Además del centro de interés, el proyecto de trabajo aborda la indagación como una estrategia de aprendizaje que genera el interés y la investigación empírica en el alumnado (Marrón Gaite, 2011, p. 114). Precisamente, Brubacher, Case y Reagan (2005) destacan la indagación como un 
procedimiento en la reflexión del docente. Y, en los alumnos, la indagación proporciona interés y autonomía en el aprendizaje, cuestiones estrechamente relacionadas con el enfoque constructivista.

\section{Los escenarios geográficos en el aprendizaje basado en problemas}

El enfoque constructivista muestra sus postulados próximos a la metodología del aprendizaje basado en problemas (ABP), tal como demuestran Savery y Duffy (2001). La autonomía y el compromiso en el alumnado, así como la intervención del docente en el proceso de enseñanza-aprendizaje resultan ser relevantes características para el desarrollo este enfoque metodológico.

El aprendizaje basado en problemas proporciona al profesor, un rol de mediador en los aprendizajes y el alumno adquiere un papel destacado en la autonomía de su aprendizaje. Además, la definición del escenario geográfico adquiere una gran importancia de cara a definir los contenidos y su organización. Así, la mediación y autorregulación del aprendizaje, junto a la extracción real de los escenarios geográficos definen esta metodología. (García De La Vega, 2010b, p. 49).

Además, la adquisición de la autonomía en el alumno para llevar a cabo el descubrimiento de los propios aprendizajes ofrecidos en el escenario geográfico se origina a partir de los conocimientos previos. Por ello, resulta relevante la actuación del profesor para flexibilizar y dinamizar la agrupación del alumnado y así alcanzar mayor interacción social y, por tanto, promover el aprendizaje entre iguales.

Los escenarios geográficos proporcionan las situaciones problema. Estas situaciones estimulan la comprensión de conceptos y proposiciones, además de conducir hacia el análisis y la asociación (Castelar y Vilhena, 2010, p. 49). Estos procesos cognitivos constituyen los pilares fundamentales del $\mathrm{ABP}$, sin dejar de lado el compromiso ético desde la valoración de la problemática de los escenarios geográficos reales. Del aprendizaje basado en problemas hay diversas posibilidades estratégicas que pudieran ser consideradas, como la indagación y la investigación.

\section{La alfabetización cartográfica: ¿estrategia didáctica o recursos educativos?}

Antes que Darwin tuviera el cometido de realizar un viaje como naturalista en el Beagle, el capitán del bergantín, Fitz Roy, ya había comenzado la cartografía de las costas de Tierra de Fuego. En el siglo XX, la colonización y dominio británico en Australia supuso la asignación sistemática de una toponimia occidental y, consecuentemente, de una transformación territorial. En cuatro meses, el capitán James Cook dio nombre a todo elemento geográfico de las costas australianas. Carter afirma que "el descubrimiento geográfico es esencialmente un proceso lingüístico" (Schögel, 2007, p. 224).

En un ámbito educativo, por el contrario, Castelar (2010, p. 157) defiende el concepto de la "alfabetización cartográfica" como el proceso de aprendizaje geográfico. En dicho proceso, el niño se acerca al conocimiento de su calle, su barrio, su ciudad... mediante la construcción gráfica y espacial, y representada en la cartografía.

Comes (2007, p. 147) vuelve a destacar el uso cotidiano de la cartografía en las aulas, considera este recurso indispensable para la observación directa, siempre que no nos quedemos en la elabo- 
ración exhaustiva de los mismos. Así esta misma autora Comes (2007, p. 149) resuelve que incluso unos esquemas gráficos permiten establecer modelos más expresivos en la construcción del conocimiento, desde la dimensión cognitiva.

Gersmehl (2008, p. 98) explora el modo de desarrollar el pensamiento espacial y desarrolla distintas actividades apoyándose en una colección de imágenes editadas en soporte digital. De esta manera, el trabajo sistemático en mapas y planos, fotografías y presentaciones renueva un trabajo proporciona una aplicación práctica a las imágenes y a las reproducciones cartográficas.

De hecho, el conciso reconocimiento de un lugar por medio de la cartografía, e incluso, de la fotografía de satélite no termina por resolver un determinado problema geográfico aunque, probablemente, proporcione estrategias para alcanzar su solución. Así, la elaboración de modelos digitales de terreno de aquellos rincones naturales desconocidos, o bien, impenetrables, permite plantear y resolver cuestiones medioambientales y relativas a la gestión del territorio imposibles hasta el momento. Sin embargo, Meirieu (2001, p.112) se plantea la coherencia del uso de las herramientas, por extensión de los recursos, sin saber las finalidades planteadas y los contenidos movilizados.

Estas técnicas debieran fortalecerse con otras habilidades en la lectura cartográfica y en la elaboración de sencillos esquemas, figuras y cartogramas, acompañados de una leyenda o cartela que evite cubrir la información representada. Estos mismos pasos de la didáctica en Geografía guían el interés de la didáctica y del currículo hacia el dominio de habilidades sobre los recursos didácticos propios de la disciplina geográfica. Precisamente, la Geografía que generará unas estrategias didácticas, previo dominio de los conceptos y destrezas geográficas, así como un planteamiento de compromiso con el territorio.

\section{CONSIDERACIONES FINALES}

El currículo de Geografía se ha modificado atendiendo a los numerosos paradigmas que se han sucedido a lo largo del siglo XX. Así, la amalgama de paradigmas educativos se muestra próxima al desarrollo epistemológico de la didáctica de la Geografía. Más aún, por cuanto que los propios paradigmas de la Geografía como ciencia académica han influido en el diseño curricular.

La propuesta del trabajo estriba en definir los elementos que constituyen el discurso epistemológico de la didáctica de la Geografía. Seguramente, alguno de los paradigmas mencionados en el artículo no han sido revisados desde una perspectiva geográfica. La discusión sobre los contenidos, y de ellos los conceptos, aún merece un seguimiento sobre la selección de los mismos y la secuencia temporal. Los métodos de trabajo en la didáctica de la Geografía tienen en común, desde hace un par de décadas, el enfoque constructivista. Tanto las experiencias desde los proyectos de investigación como desde los proyectos de trabajo realizados y vinculados con esta área de conocimiento apuntan en ese sentido. El aprendizaje basado en problemas desarrolla un campo metodológico propio, ofreciendo estrechas relaciones con el enfoque constructivista. 


\section{BIBLIOGRAFÍA}

Aisenberg, B. (1998). Sobre la programación de la enseñanza de las Ciencias Sociales. En Aisenberg, B. y Alderoqui, S. (comp.). Didáctica de las ciencias sociales II. Teorías con prácticas. Buenos Aires: Paidós, 43-76.

Audigier, F. (1992). Pensar la geografia escolar. Un repte per la didàctica. Documents d'Anàlisi Geográfica, (21), 15-33.

Bachelard, G. (2003). A formação do espirito científico, São Paulo: Contraponto.

Ball, D. L.; Thames, M. H. y Phelps, G. 2008. Content Knowledge for Teaching. What Makes It Special? Journal of Teacher Education, 59 (5), 389-407.

Batllori, R. y Serra J. M. (2010). El proyecto «Ciutat, territorio, paisatge»: un recurso innovador para la educación del paisaje en la enseñanza secundaria, Íber, (65), 17-26.

Beane, J. A. (2005). La integración del currículum, Madrid: Morata y Ministerio de Educación y Ciencia.

Benejam, P. (1992). La didàctica de la geografia des de la perspectiva constructivista, Documents d'Anàlisi Geográfica, (21), 35-52.

Brubacher, J. W.; Case, Ch. W. y Reagan, T. G. (2005). Cómo ser un docente reflexivo. La construcción de una cultura de indagación en las escuelas, Barcelona: Gedisa.

Callai, H. C. (2002). Ensino de Geografia. Práticas e textualizações no cotidiano, Porto Alegre: Mediação.

Camilloni, A. R. W. (1998). Sobre la programación de la enseñanza de las Ciencias Sociales, 183219. En Aisenberg, B. y Alderoqui, S. (Comp.). Didáctica de las ciencias sociales II. Teorías con prácticas. Buenos Aires: Paidós.

Castelar, S. V. (2011). Mudanças na prática docente. A aprendizagem em espaços não formais, PP. 69-92. En: Rego, N; Castrogiovanni, A. C. y Kaercher, N. A. (orgs.): Geografia: Práticas pedagógicas para o ensino médio, 2, Porto Alegre: Ardmed.

Castelar, S. V. y Vilhena De Moraes, J. (2010). Ensino de Geografia, São Paolo: Cengage Learning.

Castiglioni, B. (2010). La experiencia educativa en el paisaje. El proyecto 3KCL, Íber, (65), 44-55.

Cavalcanti, L. de S. (2008). A Geografia escolar e a cidade. Ensaios sobre o ensino de Geografia para a vida urbana. São Paolo: Papirus.

Cavalcanti, L. de S. (2010). Geografia, escola e construção de conhecimentos, São Paolo: Papirus, $\left(14^{a}\right.$ ed.), (1 $1^{\text {a }}$ ed., 1998).

Cavalcanti, L. de S. (2011). Ensinar geografia para a autonomia do pensamento: o desafio de superar dualismos pelo pensamento teórico crítico, Revista de ANPEGE, 7(1), 193-203. 
Chervel, A. (1988). L'histoire des disciplines scolaires. Réflexions sur un domaine de recherche, Histoire de l'Éducation, (38), 59-119. Trad. Cast.: Historia de las disciplinas escolares. Reflexiones sobre un campo de investigación, Revista de Educación, (295), 59-111.

Chevallard, Y. (1991). La transposición didáctica. Del saber sabio al saber enseñado, Buenos Aires: Aique.

Chomsky, N. (2001). La (Des) Educación, Barcelona: Crítica.

Cole, J. P. y Beynon, N. J. 1980. Iniciación a la Geografia, Fontanella, Barcelona.

Coll Salvador, C. (1997). Aprendizaje escolar y construcción del conocimiento, Barcelona: Paidós, (3 reimpresión).

Fernández Caso, M. V. (2007). Discursos y prácticas en la construcción de un temario escolar en geografía, 17-36. En: Fernández Caso, M. V. y Gurevich, R. (2007). Geografia: nuevos temas, nuevas preguntas. Buenos Aires: Biblos.

Fernández Caso, M. V. (2011). Imaginarios culturales, valoraciones sociales y selección de los contenidos en Geografía, 45-58. En Cavalcanti, L. de S.; Bueno, M. A. y Souza, V. C. de (orgs.): Produção do conhecimento e pesquisa no ensino da Geografia, Goiânia: Ed. Da PUC Goiás.

García De La Vega, A. (2004). El itinerario geográfico como recurso didáctico para la valoración del paisaje, Didáctica Geográfica, (6), 2ª época, 79-95.

García De La Vega, A. (2009). Transposición didáctica y disciplinariedad. Dos paradigmas yuxtapuestos y complementarios para una educación integral desde la didáctica de la Geografía, Actas del X Congreso Internacional de Educación, Camagüey, Cuba.

García De La Vega, A. (2010a). El desarrollo curricular a través de las áreas instrumentales, Actas del II Congrés Internacional de Didàctiques. L'activitat del Docent: Intervenció, Innovació, Investigació, Girona. En: http://hdl.handle.net/10256/2892

García De La Vega, A. (2010b). Aplicación didáctica del aprendizaje basado en problemas al análisis geográfico, Revista de Didácticas Especificas, (2), 43-60. En: http://www.didacticasespecificas.com/ files/download/2/articulos/17.pdf, consultado el 10 de octubre de 2011.

García De La Vega, A. (2011). "El paisaje: un reto curricular y didáctico", Revista de Didácticas Especificas, (4), 1-19. En: <http://www.didacticasespecificas.com/files/download/4/articulos/35.pdf> consultado el 25 de octubre de 2011 ..

García De La Vega, A. (en prensa). Aproximación al aprendizaje desde escenarios geográficos reales. Una posibilidad didáctica, Didáctica Geográfica.

García De La Vega, A. (en revisión). Estrategias innovadoras en la didáctica de la Geografía, en L. de Souza Cavalcanti (coord.): La Formação de professores: pesquisa e prática pedagógica no ensino de Geografia”, Goiânia: Universidade Estadual de Goiânia. 
García Pérez, F. F. (2011). La enseñanza de la Geografía y sus posibilidades en el currículum, 9-17. En Tonini, I. M.; Goulart, L. B.; Militz, R. E.; Castrogiovanni, A. C. y Kaercher, N. A. (Orgs.): O ensino da Geografia e suas composições curriculares, Porto Alegre: Universidade Federal do Rio Grande do Sul.

Germeshl, Ph. (2008). Teaching Geography, New York: The Guilford Press, (2ª ed.).

Gudmundsdóttir, S. y Shulman, L. S. (2005). Conocimiento Didáctico en Ciencias Sociales, Revista de currículum y formación de profesorado, 9 (2), 1-12.

Gurevich, R. (2006). Un desafío para la Geografía: explicar el mundo real, pp. 63-84. En Aisenberg, B. y Alderoqui, S. (Comp.). Didáctica de las ciencias sociales I. Aportes y reflexiones. Buenos Aires: Paidós, $12^{\mathrm{a}}$ reimpresión, (1 $\left.{ }^{\mathrm{a}} \mathrm{ed}, 1993\right)$.

Herrero Fabregat, C. (1995). Geografia y Educación, Madrid: Huerga Fierro.

Libâneo, J. C. (1992). Adeus professor, adeus professora? Novas exigências educacionais e profissão docente, São Paolo: Cortez, (12a ed., 2010).

Marrón Gaite, M. J. (2011). Enseñar y aprender Geografía a partir de distintos lenguajes. Una experiencia de trabajo por proyectos para estudiar el clima de España a partir de los refranes, Anales de Geografia de la Universidad, 31 (1), 107-123.

Meirieu, Ph. (2001). La opción de educar, Barcelona: Octaedro.

Novak, J. D. (1988). El constructivismo humano: hacia la unidad en la elaboración de significados psicológicos y epistemológicos, 23-40. En: Porlán, R.; García, J. E. y Cañal, P. (comp.): Constructivismo y enseñanza de las ciencias, Sevilla: Díada.

Oliveira, K. A. T. de. (2011). A cidade como um saber do professor de Geografia, 51-70. En: De Morais y Cavalcanti, L. DE S. (orgs.): A cidade e seus sujeitos, Goiânia: Vieira.

Perrenoud, Ph. (1998). La transposition didactique à partir de pratiques : des savoirs aux compétences, Revue des Sciences de l'éducation, vol. 24, nº 3, pp. 487-514.

Perrenoud, Ph. (2000). Le rôle de la formation à l'enseignement dans la construction des disciplines scolaires, Éducation et francophonie, 28 (2), 74-98.

Perrenoud, Ph. (2004). Desarrollar la práctica reflexiva en el oficio de enseñar, Barcelona: Graó.

Reboul, O. (1994). Introduction à la rhétorique. Théorie et pratique, Paris : Presses Universitaires de France.

Rodríguez Lestegás, F. (2000). La elaboración del conocimiento geográfico escolar: ¿de la ciencia geográfica a la geografía que se enseña o viceversa?, Íber, (24), 107-116.

Rodríguez Lestegás, F. (2007). El problema de la transposición en la enseñanza de la Geografía: ¿Y si la transposición fuese el problema?, 527-536. En: Ávila Ruíz, R. M.; López Atxurra, R. y 
Fernández De Larrea, E. (eds.). Las competencias profesionales para la enseñanza-aprendizaje de las Ciencias Sociales ante el reto europeo y la globalización, Bilbao: Asociación Universitaria de Profesores de Didáctica de las Ciencias Sociales.

Saito, C. H. (2006). Probio. Educación Ambiental, Brasilia: Universidade de Brasilia.

Savery, J. R. y Duffy, Th. M. (2001). Problem-based Learning. An instructional model and its constructivist framework, Centre for Research on Learning and Technology, Technical Report, (16)1, Bloomington: Indiana University. En: <http://www.dirkdavis.net/cbu/edu524/resources/Problem\%20 based\%201earning\%20An\%20instructional $\% 20$ model $\% 20$ and $\% 20$ its $\% 20$ constructivist $\% 20$ framework.pdf> consultado el 04 de noviembre de 2011.

Schlögel, K. (2007). En el tiempo leemos el tiempo, Madrid: Siruela.

Shön, D. A. (1992). La formación de profesionales reflexivos. Hacia un nuevo diseño de la enseñanza y el aprendizaje en las profesiones, Barcelona: Paidós.

Shön, D. A. (1998). El profesional reflexivo. Cómo piensan los profesionales cuando actúan, Barcelona: Paidós.

Shulmann, L. S. (2005). Conocimiento y enseñanza: Fundamentos de la Nueva Reforma, Revista de currículum y formación de profesorado, 9 (2), 1-30. Texto original (1987): "Knowledge and Teaching: Foundations of the New Reform", Harvard Educational Review, 57 (1), 1-22.

Souza, V. C. de. (2011). A formação acadêmica do professor de geografia: dimensões teóricas, 121142. En: Callai, C. H. (orgs.): Educação geográfica. Reflexão e Prática, Coleção de Ciências Sociais, 2, Ijuí: Editora Unijuí.

Stauffacher, M; WalteR, A.; Lang, D. J.; Wiek, A. Y Scholz, R.W. (2006). Learning to research environmental problems from functional socio-cultural constructivism perspective. The transdisciplinary case study approach, International Journal of Sustainability, 7 (3), 252-275.

Trepat, C. A. y Comes, P. (2007). El tiempo y el espacio en la didáctica de las ciencias sociales. Barcelona: Graó e Institut de Ciències de 1'Educación de la Universitat de Barcelona, $6^{\mathrm{a}}$ edición, $\left(1^{\mathrm{a}} \mathrm{ed}\right.$, 1998).

Unwyn, T. (1995). El lugar de la Geografia, Madrid: Cátedra.

Vilhena De MORAES, J. (2010). A teoria de Ausubel na aprendizagem do conceito de espaço geográfico, 97-112. En: Castelar, S. V. (org.): Educação geográfica. Teorias e práticas docentes, São Paolo: Contexto.

Vygotsky (2003). El desarrollo de los procesos psicológicos superiores, Barcelona: Crítica, (1 $\left.{ }^{\mathrm{a}} \mathrm{ed}, 2000\right)$.

Vygotsky (2010). Pensamiento y lenguaje, Barcelona: Paidós, (1ª ed. 1995).

Artículo recibido 20 - 09 - 11. Aprobado 25 - $11-11$. 